Revue des patrimoines

\title{
Chevaux travaillés et chevaux qui travaillent: réflexions sur la notion de travail dans l'univers équestre
}

Léa de Boisseuil

\section{OpenEdition}

Journals

Édition électronique

URL : http://journals.openedition.org/insitu/12101

DOI : 10.4000/insitu. 12101

ISSN : 1630-7305

Éditeur

Ministère de la culture

Référence électronique

Léa de Boisseuil, «Chevaux travaillés et chevaux qui travaillent : réflexions sur la notion de travail dans I'univers équestre », In Situ [En ligne], 27 | 2015, mis en ligne le 02 novembre 2015, consulté le 19 avril 2019. URL : http://journals.openedition.org/insitu/12101 ; DOI : 10.4000/insitu.12101

Ce document a été généré automatiquement le 19 avril 2019

\section{(c) (i) () $\Theta$}

In Situ Revues des patrimoines est mis à disposition selon les termes de la licence Creative Commons Attribution - Pas d'Utilisation Commerciale - Pas de Modification 4.0 International. 


\title{
Chevaux travaillés et chevaux qui travaillent : réflexions sur la notion de travail dans l'univers équestre
}

\author{
Léa de Boisseuil
}

2 La question, si elle peut paraître rhétorique, n'en est pas moins nécessaire. Pour repérer, archiver, protéger, valoriser un patrimoine, encore faut-il s'entendre sur les réalités qu'il englobe. Or la difficulté majeure de ce questionnement réside dans le mot travail. La grande complexité de cette notion, des réalités qu'elle désigne, des débats qu'elle soulève, rend l'approche de notre sujet d'autant plus intéressante. Ainsi, pour amorcer les discussions et réflexions autour du patrimoine du cheval au travail, faisons un petit détour théorique et historique. L'évolution des mots et de leurs usages nous permet bien souvent de saisir des enjeux contemporains. Celle du travail est, nous allons le voir, particulièrement instructive.

\section{Histoire lexicale}

Le terme de «travail » prendrait ses origines dans un mot latin, tripalium, désignant un instrument de torture à trois pieux. Cette étymologie est toutefois contestée par certains linguistes qui y voient la projection contemporaine d'une certaine vision du travail. En comparant les différentes formes que prend ce terme dans les langues indo-européennes, ils proposent des pistes différentes, notamment celle du latin trabes (poutre) ${ }^{1}$. Cette querelle étymologique, loin d'être résolue, nous fait tout de suite entrevoir les enjeux que contient cette notion de travail. Elle repose en particulier sur le fait que le latin et le grec anciens n'en proposent aucun terme équivalent. Est-ce à dire que les Grecs et les Romains ne travaillaient pas? Nous y reviendrons...

4 Si l'étymologie ne nous est pas d'un grand secours, l'histoire du mot dans la langue française nous apprend en revanche bien des choses. L'évolution sémantique révèle des 
évolutions culturelles et sociales et peut nous offrir des pistes de réflexion fécondes pour la question qui nous intéresse.

5 Le mot «travail» apparaît donc dans la langue française au $\mathrm{XII}^{\mathrm{e}}$ siècle pour désigner d'abord l'état d'une personne qui souffre, et plus spécifiquement les souffrances de l'accouchement. Progressivement, le terme va englober toute activité demandant de gros efforts et aboutissant à une transformation ou une création. On l'applique ainsi très tôt à la discipline équestre, et dès le XIV siècle, «travailler un cheval » signifie le soumettre à des séries d'exercices. Ce n'est qu'au $\mathrm{XVI}^{\mathrm{e}}$ siècle que le terme commence à désigner une activité quotidienne de subsistance, avec ses implications économiques et sociales. Mais dans le même temps, son emploi se diversifie et on parle alors du travail comme d'un processus d'élaboration ou de transformation d'une matière, d'un objet ou d'une personne, sous l'effet de l'activité humaine, d'une force naturelle ou d'une machine. Du travail du temps aux travaux d'aiguille, le mot s'enrichit donc de toute une gamme de phénomènes se référant à l'action, la transformation et la création. C'est à cette même période que l'on commence à employer ces termes (travail et travailler) pour qualifier les activités, utiles à l'homme, auxquelles on soumet les animaux domestiques. Finalement, ce n'est qu'avec le développement de la société industrielle aux XVIII ${ }^{\mathrm{e}}$ et XIX ${ }^{\mathrm{e}}$ siècles que le mot se met à désigner l'activité humaine productive et économique, socialement et politiquement organisée ${ }^{2}$.

6 Cette longue série de transformations aboutit aujourd'hui à des termes qui recouvrent des significations multiples. Outre le travail de l'accouchement et celui servant de dispositif de contention utilisé pour ferrer ou soigner bœufs et chevaux, le travail désigne de façon générale un ensemble d'activités coordonnées en vue de l'élaboration d'un ouvrage. Ce n'est que dans un sens plus spécifique qu'il s'applique aux tâches laborieuses, productives et rémunérées formant un ensemble structuré d'activités économiques. Pour finir de complexifier les choses, le terme "travail» s'emploie indifféremment pour qualifier l'ensemble des tâches et activités, la manière dont celles-ci sont effectuées, et le résultat qu'elles produisent.

7 Il paraitt important d'insister sur cette polysémie, héritée d'une histoire complexe. Car on a tendance aujourd'hui à ne plus considérer que la dimension laborieuse et économique $\mathrm{du}$ travail. Quand on dit que quelqu'un travaille, on entend seulement l'activité rémunératrice qui lui permet de subsister, toutes les autres nuances de sens ayant tendance à passer pour des utilisations imagées du mot. C'est déjà ce que remarquait au début du Xx ${ }^{e}$ siècle le philosophe Herbert Marcuse :

La notion économique du travail [...] a orienté les conceptions touchant le sens et l'essence du travail dans une direction bien déterminée, au point que maintenant, au premier chef, au sens propre du mot, c'est l'activité économique qui est considérée comme travail. L'activité du politicien, du chercheur, de l'artiste, du prêtre, n'est prise comme travail qu'au sens figuré et non sans hésitation³

Et il ajoute plus loin: «De plus en plus, on a tendance à ne plus entendre par là que le travail d'exécution, le travail commandé, non libre, dont le travail de l'ouvrier salarié est le prototype ${ }^{4} »$. L'histoire du mot nous apprend pourtant que le travail comme activité rémunératrice est apparu tardivement, et que le travail hors de la sphère strictement économique, loin d'être un dérivé figuratif du terme, en est au contraire le sens originel. 


\section{Cheval de travail versus cheval au travail}

9 Toutes ces nuances de sens ont leur intérêt pour répondre à notre question: qu'est-ce qu'un cheval au travail ? Il faut distinguer ce que désigne le terme de " cheval au travail », du terme «cheval de travail ». Ce dernier désigne généralement un cheval de trait, de labour, de poste, de mine, etc., en tout cas un animal utilisé pour sa force motrice et qui collabore à l'activité économique de l'homme, activité perçue comme laborieuse. Le terme de «cheval au travail» quant à lui, semble ouvrir le champ à un panel plus large d'utilisations. Et cet élargissement de la notion de travail dans la relation de l'homme et du cheval convient davantage aux réalités équestres.

10 En effet, dans tous les domaines équestres, y compris ceux du sport et du loisir, des professionnels utilisent des chevaux dans le cadre de leurs activités commerciales ou d'enseignement. Les chevaux de club, d'instruction, de randonnée sont alors des chevaux impliqués dans la sphère économique du travail. Sans être généralement assimilés à des chevaux de travail, ils sont toutefois des chevaux au travail ${ }^{5}$. De façon un peu différente, un cheval de sport, sans nécessairement faire l'objet d'un projet économique, peut lui aussi être considéré comme un «cheval au travail». Du moins est-il un cheval qui travaille, que l'on fait travailler et que l'on travaille... Et il en va ainsi de tous les chevaux utilisés dans le cadre d'une pratique équestre de loisir. Car tous les cavaliers amateurs, sans objectifs économiques et selon leurs propres termes, travaillent et font travailler leurs chevaux. Le cheval de chasse n'échappe pas à la règle puisque les veneurs affirment qu'en dehors des saisons de chasse, leurs bêtes restent au pré et « ne travaillent pas ${ }^{6}$ ».

11 Ces expressions cavalières, entendues aux détours des écuries, qui évoquent sans cesse le travail équestre, ne sont pas seulement des expressions imagées mais comportent un sens littéral. Elles définissent les relations entre le cavalier et sa monture. Relations qui se déploient dans des "activités d'élaboration et de transformation visant un but", sens premier du mot travail. Ce but pouvant être l'acquisition d'un dressage particulier, l'amélioration de performances sportives, l'accomplissement de prouesses techniques ou motrices, ou encore le renforcement de la relation elle-même...

12 En parlant de travail dans le domaine équestre, on fait donc référence à des réalités multiples et complexes, qu'il n'est pas aisé de définir, ni de circonscrire. Quand on parle de «cheval au travail», comment en effet distinguer le travail du cheval pour servir l'homme, du travail de l'homme sur le cheval? De même, faut-il considérer les chevaux comme les objets, les instruments ou les acteurs du travail équestre ? Quelle différence y a-t-il entre un cheval qui travaille, un cheval que l'on fait travailler, et un cheval que l'on travaille?

13 C'est volontairement que l'énumération de ces questionnements brouille quelque peu mon propos. Car elle a vocation à montrer à quel point cette question du travail dans la relation du cavalier et du cheval est complexe et par conséquent essentielle. Ainsi, dans l'expression « les chevaux au travail » faut-il seulement entendre les chevaux « dans » la sphère économique et sociale du travail ? Ou bien plutôt élargir nos conceptions à l'ensemble des chevaux dont les cavaliers élaborent pour eux des projets de perfectionnement, de transformation, etc.? Car la notion de travail, dans le cadre d'activités équestres, va bien au-delà de la stricte sphère économique qui associe le travail à l'emploi rémunéré et au labeur. On l'a vu, on parlait déjà de travail équestre avant que le terme ne désigne des activités économiques de subsistance. Le travail n'est-il pas 
finalement le fondement même de la relation si particulière que nous entretenons avec les chevaux, relation tout en nuances comme l'est également cette notion de travail ?

Car enfin, s'il y a d'autres animaux qui travaillent (comme les chiens policiers, les chiens de berger, etc.), d'autres animaux impliqués dans la sphère économique du travail (animaux de rente, animaux de zoos ou de cirques), il n'y a pas à ma connaissance d'autres animaux que l'on travaille et qui sont l'ouvrage même que l'on cherche à élaborer. A-t-on jamais entendu un propriétaire de chien, de chat ou de cochon, dire qu'il doit «travailler » son animal comme on travaille une matière, et comme on travaille un cheval?

\section{La menace du travail}

Ainsi, au regard de l'histoire sémantique, il semble pertinent d'élargir nos conceptions pour éviter de réduire «le cheval au travail » à une activité productive et laborieuse. Il convient au contraire de prendre la mesure de ce vaste ensemble de réalités équestres qui place le travail, avec toutes ses nuances, au cœur de la relation de l'homme et du cheval. Et cette affirmation selon laquelle « le cheval au travail » ne peut se réduire à un animal soumis à une activité de labeur semble d'autant plus nécessaire qu'elle est justement à l'origine des revendications portées par des associations de protection animale. Celles-ci ne perçoivent dans la relation de travail que la souffrance et l'aliénation que l'homme ferait subir à ses animaux, et entendent donc les abolir.

L'actualité fourmille d'exemples qui viennent appuyer ce constat. À New York, par exemple, des associations (PETA, NYClass), soutenues par le nouveau maire Bill de Blasio ${ }^{7}$, tentent de faire interdire les calèches qui sillonnent Manhattan depuis deux siècles. Pour ces militants, faire vivre et travailler des chevaux en milieu urbain est une aberration :

47 semaines par an, ces animaux sont privés de la liberté et de la possibilité de vivre comme des chevaux. Ils travaillent 9 heures par jour, 7 jours par semaine, et vivent dans des boxes deux fois plus petits que ce qu'ils devraient être. [...] Il est difficile de ne pas reconnaître la souffrance de ces chevaux. Exploités pour divertir et enrichir l'humain, le cheval d'attelage new-yorkais est une icône de la misère ${ }^{8}$.

On décèle bien dans cet argumentaire le prototype du travail ouvrier et industrieux qu'évoquait $\mathrm{H}$. Marcuse, qui sous-entend une soumission contrainte à une activité laborieuse, productrice de souffrance et d'aliénation. L'invocation de l'essence profonde du cheval, en tant qu'être libre et naturel, revient constamment dans les propos de ces associations. En outre, que cette relation entre hommes et animaux, ici entre cochers et chevaux, soit associée à un projet économique, et donc, dans l'esprit des associations, à une visée strictement utilitariste, représente pour elles une abomination éthique et morale. C'est donc en s'appuyant sur ce type d'argumentaire ${ }^{9}$ que se mobilisent militants et équipes politiques pour faire interdire cette activité touristique et folklorique qui fait vivre actuellement quelque 170 cochers et 220 chevaux new-yorkais.

Cette confrontation entre associations de protection animale et éleveurs-utilisateurs d'animaux n'est pas un cas isolé. Elle fait écho par exemple aux polémiques qui agitent le milieu du traîneau à chien au Québec depuis quelques années. Et nous profitons de cette évocation de l'espèce canine pour soulever une question: si le travail des chiens de traîneau fait l'objet de contestation, pourquoi celui des chiens de berger ne soulève-t-il pas autant d'indignation? Le labeur animal n'est-il perçu comme tel que lorsque celui-ci fait appel à des capacités physiques? La souffrance et l'aliénation ne sont-elles l'apanage 
que du travail physique et moteur, le travail intellectuel étant lui plus valorisé, et valorisant?

Mais revenons à nos chevaux. Affirmer l'idée d'un travail élargi à ses définitions originelles, qui ne se laisse pas circonscrire à sa portion économique et laborieuse, c'est donc réaffirmer les relations spécifiques de l'homme et du cheval, qui tendent aujourd'hui, comme d'autres relations de travail, à être remises en cause pour des raisons dont le fondement éthique gagnerait à davantage de réflexion.

\section{Le travail, un fait de culture}

Mais nous aimerions apporter un autre éclairage sur cette spécificité du travail du cheval, en revenant sur cette évolution du mot. Car l'histoire lexicale révèle également les trajectoires sociales, culturelles, économiques et politiques qui définissent nos sociétés occidentales.

On a vu comment l'absence de notion équivalente au travail dans les langues grecques et latines était à l'origine d'une étymologie houleuse. Il n'y avait pas dans ces sociétés de catégorie regroupant l'ensemble des activités humaines telle que celle proposée par ce mot travail. L'organisation sociale des mondes antiques reposait alors sur des catégories bien différentes des nôtres, et les activités économiques se trouvaient désignées par une variété de termes irréductibles à cette réalité englobante que peut représenter le travail ${ }^{10}$.

Des travaux en philosophie, en anthropologie et en ethnolinguistique ont donc montré que cette notion de travail, essentielle et essentialisée dans nos sociétés occidentales contemporaines, n'a rien d'universel. Il y a des sociétés sans travail, des sociétés où la langue ne contient pas de notion équivalente à notre terme "travail», où les liens sociaux, économiques et politiques ne sont pas fondés sur le travail et où le rapport de l'homme à son environnement naturel n'est pas médiatisé en priorité par le travail ${ }^{11}$.

C'est donc une spécificité culturelle de nos sociétés occidentales modernes que de voir dans le travail l'essence de l'homme et de sa place dans le milieu naturel (l'homme par le travail transforme le monde et se l'approprie), un facteur essentiel de cohésion sociale, un support de la vie collective, le mode privilégié de réalisation de soi, d'épanouissement individuel, etc. Notons qu'en regard de ces valeurs extrêmement positives, des valeurs négatives sont également associées à l'idée de travail, en particulier lorsque ces promesses ne sont pas tenues : il devient alors synonyme de souffrance et d'aliénation.

Le travail, tel que nous l'avons conçu au fil de l'histoire, représente donc un fondement important de notre identité culturelle. En définitive, nous nous définissons en tant qu'Hommes et Société en nous appuyant sur le travail. Or nous faisons y participer les animaux avec lesquels nous vivons. Cette place que nous leur accordons dans le travail, et en particulier au cheval, avec toutes les nuances que nous avons vues, n'est-elle pas alors une façon de les intégrer à notre communauté culturelle? Les relations de travail entre hommes et animaux, et plus particulièrement entre hommes et chevaux, ne sont-elles pas aussi un des fondements de notre manière d'être homme, et d'être une société, comme l'affirme par exemple Jocelyne Porcher ${ }^{12}$ ?

S'intéresser à cette notion nous amène donc à la reconnaitre comme une spécificité culturelle de notre société occidentale. À ce titre, le « cheval au travail » est bel et bien un fait de culture, qui nous définit autant que lui, et qui renforce encore cette idée selon 
laquelle les relations que nous entretenons avec lui sont bien plus que de simples associations au sein d'activités productives.

\section{Le patrimoine du cheval au travail}

Le travail cristallise aujourd'hui les plus grandes aspirations et les pires craintes, aussi bien à l'échelle individuelle que collective. Il est à la fois promesse et menace, rédemption et fléau... Les relations entre hommes et animaux, loin d'être des épiphénomènes, sont modelées par des enjeux qui les dépassent largement et s'inscrivent dans des trajectoires sociales et culturelles. À ce titre, la question du «cheval au travail » fait écho à des problématiques qui n'ont rien d'accessoire.

Dans ce contexte bouleversé, où les relations de travail entre hommes et animaux font l'objet de polémiques vives, nous pensons qu'il n'est pas inutile de s'accorder le temps de la réflexion. Ce retour sur l'histoire du mot que nous avons proposée ici n'est qu'une piste mineure, qui n'explore finalement qu'une toute petite portion de cette réalité complexe $\mathrm{du}$ «cheval au travail ». Mais elle permet déjà d'en apercevoir la richesse historique et sociale.

On parle donc bien ici d'un patrimoine culturel, d'un héritage qui a construit notre identité et qui nous définit ici et maintenant. Valoriser et protéger ce patrimoine, c'est valoriser et protéger ces relations si particulières, construites au fil de l'histoire, entre les hommes et les chevaux dans notre société. Relations qui perdurent dans des formes actualisées, et qui méritent qu'on y accorde une attention raisonnée, sans céder aux attitudes outrageusement critiques ou bienveillantes.

\section{NOTES}

1. - Pour plus d'informations et de références sur ce sujet, voir l'article de Jean-Luce Morlie publié sur le site: http://blogs.mediapart.fr/blog/jean-luce-morlie/280911/tripalium-uneetymologie-ecran-archive (consulté le 24/09/14).

2. - Sur l'évolution des mots travail et travailler voir : Dictionnaire historique de la langue française. REY, Alain (dir.). Paris : édition Le Robert, 1992, réimprimé en 2000.

3. - JUNG, Joël. Le travail. Paris : Flammarion, 2000, p. 68.

4. - Herbert Marcuse (1933), cité dans JUNG, Joël. Le travail. Paris : Flammarion, 2000, p. 68.

5. - La contribution de Catherine Tourre-Malen présente bien cet aspect du cheval au travail. METTRE LE LIEN AU CHARGEMENT SUR ARTICLE TOURRE-MALEN (FC).

6. - Ces affirmations reposent sur des enquêtes de terrain réalisées auprès de différents types de cavaliers dans le cadre d'un travail doctoral à l'Ehess.

7. - La campagne de Bill de Blasio, élu en janvier 2014, a bénéficié d'un financement non négligeable de la part de l'association locale de défense des animaux, NYClass.

8. - Voir le site : http://carriagehorsesnyc.blogspot.fr/ (consulté le 24/09/2014).

9. - Précisons que le remplacement de cette activité par des voitures vintage électriques, et la libération des immenses locaux qui abritent les écuries en plein cœur de Manhattan, ouvrent 
aussi des perspectives lucratives pour un certain nombre de groupes commerciaux qui ne sont pas étrangers au lobbying contre les calèches new-yorkaises.

10. - Voir sur ce point les travaux de VERNANT, Jean-Pierre. Mythe et pensée chez les Grecs, étude de psychologie historique. Paris : La Découverte, 2005.

11. - Voir par exemple sur cette question les travaux de MÉDA, Dominique. Le travail : une valeur en voie de disparition. Paris : Aubier, 1995 ; Travail : la révolution nécessaire. La Tour d'Aigues : Éd. de l'Aube, 2010.

12. - PORCHER, Jocelyne. « Ne libérez pas les animaux!». Revue du MAUSS, 2007, n² 29, p. 575-585 ; Vivre avec les animaux, une utopie pour le XXI ${ }^{e}$ siècle. Paris : La Découverte, 2014.

\section{RÉSUMÉS}

En revenant sur l'histoire du mot travail dans la langue française, cette contribution propose une appréciation élargie des relations de travail entre hommes et chevaux, relations qui ne peuvent se réduire aux seules activités productives et laborieuses. Les activités équestres déploient au contraire une variété de relations qui d'une manière ou d'une autre visent l'élaboration et le perfectionnement, deux aspects fondamentaux du travail. Le statut essentiellement historique et social de cette notion donne au patrimoine du «cheval au travail » son caractère profondément culturel et identitaire.

Worked horses and working horses, some thoughts on the notion of work in the equestrian world. Looking back over the history of the word 'work' in the French language, this paper proposes an enlarged appreciation of the working relationship between men and horses, relationships that cannot be reduced only to productive and working activities. On the contrary, equestrian activities generate a variety of relationships, aimed at elaboration and improvement, two fundamental aspects of work. The historical and social status of the concept of work gives a deep cultural and identity character to the heritage of the 'workhorse'.

\section{INDEX}

Mots-clés : chevaux, travail, anthropologie, animaux

Keywords : horses, work, anthropology, animals

\section{AUTEUR}

\section{LÉA DE BOISSEUIL}

Doctorante en anthropologie sociale à l'École des hautes études en sciences sociales lea.deboisseuil@gmail.com 